María Nieves Tapia*

ORCID: 0000-0002-0403-9629

Buenos Aires, Argentina

\title{
Ama y comprenderás: el carisma de Chiara Lubich en diálogo con la pedagogía del aprendizaje-servicio
}

\author{
Love and You Will Understand: \\ Chiara Lubich's Charisma in Dialogue \\ with the Pedagogy of Service-Learning
}

Summary: Having originated from very old and diverse roots, the pedagogy of service-learning continues to spread on five continents under different names. It was given its present name in the USA and has developed systematically since 1968. Two years earlier, Chiara Lubich founded the Gen Movement, a youth-based branch of the Focolare Movement. The article explores her key speeches and messages that she addressed to young people. Lubich's charisma inspired many practices

* María Nieves Tapia es graduada en Historia, con especialización en Historia Antigua de Roma. Fue profesora adjunta de la Cátedra de Historia Antigua II en la Pontificia Universidad Católica Argentina en Buenos Aires. Desde 1993 se dedica a la investigación y promoción de la pedagogía del aprendizaje-servicio. Fundó y dirigió los programas de aprendizaje-servicio del Ministerio de Educación de Argentina. Actualmente es Directora del Centro Latinoamericano de Aprendizaje y Servicio Solidario (CLAYSS, www.clayss.org/ info@clayss.org.ar), organización civil sin fines de lucro dedicada a la promoción e investigación sobre aprendizaje-servicio. Contacto: Yapeyú 283, C1202ACD Ciudad de Buenos Aires, Argentina; e-mail: info@clayss. org; ntapia@clayss.org.ar. 
and reflections on service-learning, laying the foundations for this pedagogy. They may be easier to understand if analysed through the prism of ideas discussed by Lubich in her studies, writings and works that inspired the Focolare Movement. The article presents the first reflections on the contemporary dialogue between Chiara Lubich's charisma and the pedagogy of service-learning. The first part proposes a brief introduction to the idea of pedagogy of service-learning, while the second offers an analysis of some of its fundamental features present in the dialogue with the 'charisma of unity'.

Keywords: service-learning; Chiara Lubich; pedagogy.

Abstract: La pedagogía del "aprendizaje-servicio", hoy difundida en los cinco continentes bajo diversas denominaciones, tiene raíces muy antiguas y diversas, pero tomó nombre propio en Estados Unidos y comenzó a difundirse sistemáticamente desde 1968. Dos años antes, Chiara Lubich fundaba el Movimiento Gen, la rama juvenil del movimiento de los Focolares. Los discursos y mensajes que dirigiera a los jóvenes a partir de esa fecha son una de las fuentes principales de este artículo. El carisma de Lubich ha inspirado muchas prácticas y reflexiones sobre el aprendizaje-servicio, y los principios fundamentales de esta pedagogía pueden iluminarse en diálogo con afirmaciones que Lubich hizo en sus meditaciones y escritos, y también en las acciones que impulsó dentro del Movimiento de los Focolares. Se presentará sintéticamente una primera aproximación al diálogo contemporáneo entre el carisma de Chiara Lubich y la pedagogía del aprendizaje-servicio. En la primera sección se ofrece una breve introducción a la propuesta pedagógica del aprendizaje-servicio, y en la segunda parte se profundizan algunas de sus características fundamentales en diálogo con el "carisma de la unidad".

Palabras clave: aprendizaje-servicio; Lubich; pedagogía.

El objetivo de este artículo es ofrecer una primera aproximación al diálogo contemporáneo entre el carisma de Chiara Lubich y la pedagogía del aprendizaje-servicio, presentando una síntesis del estado de la cuestión, tomando como referencia especialmente la bibliografía desarrollada en América Latina y España. En la primera sección se ofrece una breve introducción a la propuesta pedagógica del aprendizaje-servicio, y en la segunda parte se profundizan algunas de sus características fundamentales en diálogo con el "carisma de la unidad". 


\section{Una aproximación a la pedagogía del "aprendizaje-servicio"}

Recolectar ropa y alimentos para una población vulnerable es un servicio.

Estudiar arquitectura y diseñar planos es aprendizaje.

Estudiar arquitectura, diseñar planos y contribuir

a la construcción de viviendas dignas junto con una población vulnerable, es aprendizaje-servicio solidario.

Sería imposible sintetizar la vasta bibliografía e investigación contemporánea sobre la propuesta pedagógica conocida como "service-learning" o "aprendizaje-servicio", ni la complejidad de matices culturales y debates en curso. Simplemente a título introductorio señalemos que son muchas las definiciones posibles del concepto de aprendizaje-servicio: "Aprender haciendo al servicio de la comunidad"; "Reflexión y acción transformadora de la realidad"; "Servicio solidario protagonizado por los jóvenes y planificado intencionadamente con contenidos de aprendizaje"1.

Los primeros desarrollos pedagógicos sobre esta temática tienen más de 50 años, y nacen de la confluencia del pensamiento de John Dewey y el experiential learning con otras corrientes pedagógicas de la segunda mitad del siglo XX, especialmente la pedagogía crítica del brasilero Paulo Freire².

Es importante subrayar que "la historia de la práctica de aprendizaje-servicio es mucho más antigua que el término mismo", y que muchas instituciones educativas y organizaciones sociales en todo el mundo desarrollan proyectos que utilizan los aprendizajes desarrollados para ofrecer ser-

${ }^{1}$ María Nieves Tapia, Aprendizaje y servicio solidario en el sistema educativo y las organizaciones juveniles (Buenos Aires: Ciudad Nueva, 2006).

2 Paulo Freire, Myles Horton, We Make the Road by Walking: Conversations on Education and Social Change (Philadelphia: Temple University Press, 1991); Thomas Deans, „Service-Learning in Two Keys: Paulo Freire's Critical Pedagogy in Relation to John Dewey's Pragmatism", Michigan Journal of Community Service Learning 6 (1999): 15-29; Danika M. Brown, Pulling it Together: A Method for Developing Service-Learning and Community Partnerships Based in Critical Pedagogy (Washington DC: National Service Fellow Research, 2001).

3 Peter Titlebaum, Gabrielle Williamson, Corinne Daprano, Janine Baer, Jayne Brahler, Annotated History of Service Learning 1862-2002 (Dayton, OH: University of Dayton, May 2004). 
vicios concretos a la comunidad, aunque no conozcan la terminología ni la bibliografía especializada sobre "aprendizaje-servicio". Veamos unos pocos ejemplos:

- Niños del CEI Atalaya (Granada, España) aplicaron sus conocimientos de Ciencias Naturales para investigar cómo contribuir a la restauración del estado ecológico del mayor estanque de Atarfe, en su ciudad, arrastrando en la iniciativa a más de mil personas de la localidad. Lo que era una gran extensión de agua estancada volvió a ser un espejo de agua cristalina con especies autóctonas, y un gran centro de recreación y disfrute de la naturaleza para toda la ciudad ${ }^{4}$.

- En St. Mary County (Maryland, USA), los alumnos de $8^{\circ}$ grado investigaron en Historia el pasaje de la esclavitud a la emancipación de los afro-americanos, y al mismo tiempo desarrollan un proyecto de preservación de la antigua plantación esclavista Sotterley, un sitio histórico local en riesgo 5 .

- Estudiantes de escuelas técnicas de la Patagonia argentina aplican sus conocimientos para diseñar e instalar generadores eólicos e hidroeléctricos para poblaciones rurales aisladas 6 .

- En Malasia, los estudiantes de Ciencias Económicas asesoran a pequeños comerciantes de la periferia de Kuala Lumpur para que puedan mejorar sus prácticas contables, de logística y marketing para garantizar mejores ingresos a sus familias?

- Un grupo de líderes de las Guías de Francia participó junto con los Scouts en un proyecto de animación de niños y jóvenes refugiados en el África subsahariana. Aplicando lo aprendido en sus grupos, dictaron cursos de primeros auxilios, y formaron a jóvenes refugiados para que pudieran a su vez ser animadores de actividades que puedan educar a los niños refugiados a través del juego ${ }^{8}$.

${ }^{4}$ Premio Aprendizaje-servicio de España, 2015. https://youtu.be/inkxzoeHK44; https:// aprendizajeservicio.net/premio-aprendizaje-servicio-2015/. Salvo indicación en contrario, todos los enlaces han sido consultados en agosto de 2018.

${ }^{5}$ www.nylc.org, consultado octubre 2005.

${ }^{6} \mathrm{https} / / /$ youtu.be/8GLU1992gSY

7 UKM, National University of Malaysia, Strengthening Community Engagement. Nurturing Caring Citizens (Kuala Lumpur: UKM, 2012), 19.

8 Silvia Chanes, Graciana Gaona, María Nieves Tapia, "Caminos de solidaridad", Conferencia Internacional Católica del Guidismo, Documentos pedagógicos, 2006, http:// www.clayss.org.ar/04_publicaciones/Caminosdesolidaridad-esp.pdf 
Las prácticas de aprendizaje-servicio hoy se desarrollan en los cinco continentes, en todos los niveles de la educación formal como en la educación no formal y las organizaciones sociales, especialmente las que trabajan con niños y jóvenes. En diferentes contextos culturales pueden rastrearse múltiples raíces teóricas ${ }^{9}$, y también la influencia de valores y creencias propios de cada cultura. Por ejemplo, en chino se utilizan varios caracteres asociados al pensamiento de Confucio para explicitar el concepto de "aprendizaje-servicio" 10 , en India el servicio social universitario se inspira en el pensamiento de Gandhi ${ }^{11}$, y en Ecuador se fundamentaron las políticas de promoción del aprendizaje-servicio en la tradición ancestral quechua del suma kawsay o "buen vivir"12.

La pedagogía del aprendizaje-servicio responde simultáneamente a los "cuatro pilares" de la educación para el siglo XXI planteados por UNESCO: "aprender a aprender, a hacer, a ser y a vivir juntos"13, y está directamente emparentada con la mayoría de las nuevas estrategias de innovación pedagógica (el aprendizaje en base a proyectos, el aprendizaje basado en problemas, el aprendizaje por diseño y otros). Al mismo tiempo, recoge en la práctica pedagógica estrategias y formas de planeamiento propias del voluntariado y el trabajo social ${ }^{14}$.

La evidencia muestra que las buenas prácticas de aprendizaje-servicio no sólo inciden en la resolución de problemas de la comunidad, sino que impactan directamente en la calidad y la inclusión educativa. Investigaciones desarrolladas en varias partes del mundo ${ }^{15}$ han documentado los impactos

9 PaSo Joven: Participación Solidaria para América Latina, "Manual de formación de formadores en aprendizaje-servicio y servicio juvenil" (Buenos Aires, BID-SES-CLAYSSALIANZA ONG-CEBOFIL, 2004), http://www.clayss.org.ar/paso_joven/index.htm

${ }^{10}$ Office of Service-Learning, Lingnan University, Annual report 2011-2012 (Hong Kong: Lignan University, 2012).

${ }^{11}$ International Association for National Youth Service, Actas de la $6^{a}$ Conferencia Global/ Procedures of the VI Global Conference (Buenos Aires: CLAYSS, 2002).

${ }_{12}$ Aprendizaje-servicio (ApS): claves para su desarrollo en la Universidad, coord. Laura Rubio, Anna Escofet (Barcelona, Octaedro-ICE, 2017), 55-68.

13 Jacques Delors, La educación encierra un tesoro (Buenos Aires: Santillana, Ediciones UNESCO, 1996).

${ }^{14}$ Amanda Moore McBride, Carlos Benitez, Michael Sherraden, The forms and Nature of Civic Service: A Global Assessment (St Louis: Global Service Institute, Center for Social Development, Washington University in St. Louis, 2003).

${ }_{15}$ Janet Eyler, Dwight E. Giles, Where's the learning in service-learning? (San Francisco: Jossey-Bass Publishers, 1999); Alba González, Pablo Elicegui, "El impacto de los proyectos 
positivos de las prácticas de aprendizaje-servicio en la calidad educativa integral, tales como impactos en un mejor rendimiento académico (incluso medido en test estandarizados), el desarrollo de competencias para el mundo del trabajo y la participación ciudadana, el desarrollo del pensamiento crítico, la motivación para investigar, el comportamiento prosocial y otros. También hay evidencias del impacto de las buenas prácticas de aprendizaje-servicio en la inclusión y retención de las poblaciones más vulnerables en el sistema educativo. Por ello, un número creciente de políticas educativas y de instituciones de todos los niveles en los cinco continentes comienzan a incluir la promoción del aprendizaje-servicio como parte de sus esfuerzos de reforma y mejora educativa ${ }^{16}$.

de aprendizaje-servicio en la calidad educativa. Reflexiones en torno a ocho experiencias", en: Ministerio de Educación, Ciencia y Tecnología, Aprendizaje y servicio solidario. Actas del 5to. y 6to. Seminario Internacional "Aprendizaje y servicio solidario” (República Argentina, 2004), 188-206; Shelley Billig, "Heads, Hearts, and Hands: The Research on K-12 Service Learning", en: Growing to Greatness: The State of Service Learning Project 2004 Report (St. Paul: NYLC \& State Farm, 2004); Shelley Billig, "Lessons from Reseach on Teaching and Learning: Service-learning as Effective Instruction", en: Growing to Greatness: The State of Service Learning Project 2004 Report (St. Paul: NYLC \& State Farm, 2006); Andrew Furco, "Impacto de los proyectos de aprendizaje-servicio", en: Aprendizaje y servicio solidario en la Educación Superior y en los sistemas educativos latinoamericanos. Actas del 7 mo. Seminario Internacional "Aprendizaje y Servicio Solidario” (República Argentina: Ministerio de Educación, 2005), 19-26, http://www.clayss.org.ar/seminario/anteriores/actas/2005_Actas7. pdf; Andrew Furco, "The community as a resource for learning: an analysis of academic service-learning in primary and secondary education", en: The Nature of Learning. Using Research to Inspire Practice, ed. Hanna Dumont, David Instance, Francisco Benavides (Paris: Organisation for Economic Co-operation and Development, Centre for Educational Research and Innovation, 2010); Barbara Jacoby, Service-learning Essentials. Questions, Answers, and Lessons Learned (San Francisco: Jossey Bass, 2015); CLAYSS, Programa de aprendizajeservicio como política educativa nacional en Uruguay. Evaluación de resultados (Buenos Aires: CLAYSS-Porticus-Zigla, 2017).

${ }_{16}$ Roser Batlle, El aprendizaje-servicio en España: el contagio de una revolución pedagógica necesaria (Madrid: PPC, 2013); Rodrigo Mardones, Educación y fraternidad: Un principio para la formación ciudadana y la convivencia democrática (Buenos Aires: Ciudad Nueva, 2013); Enrique Ochoa, "Aprendizaje-servicio en América Latina. Apuntes sobre pasado y presente", TZHOECOEN 5 (2010): 108-125; María Nieves Tapia, Enrique Ochoa, "Legislación y normativa latinoamericana sobre servicio comunitario estudiantil y aprendizajeservicio", en: Actas de la III Jornada de investigadores sobre aprendizaje-servicio, comp. María Alejandra Herrero, María Nieves Tapia (Buenos Aires: CLAYSS-Red Iberoamericana de aprendizaje-servicio, 2015), 91-96. 


\section{Un diálogo entre la pedagogía del aprendizaje-servicio y el carisma de Chiara Lubich**}

En las últimas dos décadas, varios autores iberoamericanos hemos puesto en diálogo la pedagogía del aprendizaje-servicio con el carisma ${ }^{17}$ de Chiara Lubich, como una contribución al desarrollo de los fundamentos teóricos y metodológicos del concepto de "aprendizaje-servicio solidario". En el breve espacio de este artículo intentaremos sintetizar algunas reflexiones surgidas de este diálogo, especialmente en torno al concepto de "aprendizaje-servicio solidario" (AYSS), que se define en torno a tres ejes fundamentales:

1. Acciones de servicio solidario destinadas a atender en forma acotada y eficaz necesidades reales y sentidas, junto con una comunidad, y no sólo para ella.

** Esta sección sintetiza algunas de las reflexiones surgidas del equipo de CLAYSS y especialmente las expuestas en las siguientes obras de miembros del equipo de CLAYSS que también forman parte o están cercanos al Movimiento de los Focolares: Graciela del Campo, Protagonismo estudiantil solidario: alternativas de participación ciudadana y compromiso politico desde la escuela. La experiencia de estudiantes que participan de Proyectos educativos solidarios (Ponencia presentada en: Universidad Nacional de Avellaneda, Primeras Jornadas de Educación y Política, 24 de septiembre de 2013); Graciela del Campo, La educación solidaria en el marco de la nueva evangelización de lo social. Tesis de Maestría en Doctrina Social de la Iglesia (Madrid: Universidad Pontificia de Salamanca, inédito, 2015); Congregation for Catholic Education, Educating today and tomorrow. A renewing passion (Vatican City, 2014); Mardones, Educación; María Nieves Tapia, La solidaridad como pedagogía (Buenos Aires: Ciudad Nueva, 2000); Nieves Tapia, Aprendizaje; María Nieves Tapia, Gerardo Bridi, María Paula Maidana, Sergio Rial, El compromiso social como pedagogía. Aprendizaje y solidaridad en la escuela (Bogotá: CELAM, 2015). Ver también el manual de uso interno desarrollado por el Movimiento "Chicos por un Mundo Unido" de los Focolares [Movimento dei Focolari, "Sei tappe per un obiettivo", Teens (2018), http://www.teens4unity.org/wp-content/uploads/20186x1-AgendaCs6_ITA-.pdf].

17 Utilizamos intencionadamente el término "carisma" - en el pensamiento cristiano, un don concedido por Dios a algunas personas en beneficio de toda la comunidad- y no el término quizás más frecuente en el ámbito académico de "pensamiento", porque entendemos que una comprensión adecuada del legado de Chiara Lubich exige el análisis no sólo de sus textos, sino de su praxis como educadora de generaciones de jóvenes y adultos, y de la inseparable relación entre su vida y el "carisma de la unidad", entre su persona y el movimiento de los Focolares, del que alguna vez dijo: "nuestro movimiento y nuestra historia pueden ser considerados como un gran, extraordinario evento educativo" [Chiara Lubich, Una cultura nueva para una nueva sociedad. Doctorados honoris causa-Congresos Internacionales (1996-2001) (Buenos Aires: Ciudad Nueva, 2003), 175]. 
2. protagonizadas activamente por los estudiantes desde el planeamiento a la evaluación,

3. articuladas intencionadamente con los contenidos de aprendizaje (contenidos curriculares, reflexión, desarrollo de competencias para la ciudadanía y el trabajo, investigación) ${ }^{18}$.

\subsection{Una solidaridad fraterna y "horizontal"}

Sin duda uno de los temas más debatidos a nivel mundial en el campo del aprendizaje-servicio es el alcance y significado del término "servicio". Como señalan dos especialistas sudafricanas:

Tenemos que elegir activamente entre un aprendizaje-servicio como beneficencia $^{19} \mathrm{y}$ un aprendizaje-servicio como cambio social. [...] El cambio social está estrechamente alineado con un programa de justicia social cuyo fin es trabajar hacia una sociedad en la que individuos y grupos accedan a un trato equitativo y una justa participación en las oportunidades y beneficios de la sociedad ${ }^{20}$.

En la perspectiva de CLAYSS ${ }^{21}$ y otras organizaciones iberoamericanas $^{22}$, la opción por el aprendizaje-servicio como cambio social se expresa justamente en el concepto de "servicio solidario", y en su diferenciación con otras formas de servicio.

La inclusión del término "solidario" junto al de "servicio" pretende asociar las prácticas de aprendizaje-servicio a la construcción de vínculos interpersonales solidarios y fraternos, y definir más precisamente el término "ser-

18 CLAYSS, Guía para desarrollar proyectos de aprendizaje-servicio solidario. Edición Perú (Lima: Ediciones CLAYSS, 2018), 30-39, http://www.clayss.org.ar/04_publicaciones/ Manual_Peru_para_web.pdf

${ }_{19}$ En el original inglés se utiliza el término "charity". A los efectos de este artículo hemos preferido usar el término que consideramos más preciso de "beneficencia". Para los cristianos "Deus Caritas est", y por lo tanto entendemos que el verdadero amor o caridad cristiana no puede ser identificado exclusivamente con la limosna o beneficencia.

${ }^{20}$ Service-learning in South Africa, ed. Ruksana Osman, Nadine Petersen (Cape Town: Oxford University Press, 2013), 9.

${ }^{21}$ Centro Latinoamericano de Aprendizaje y Servicio Solidario, www.clayss.org.

${ }^{22}$ Agrupadas en la Red Iberoamericana de aprendizaje-servicio, REDIBAS, http://www. clayss.org.ar/redibero.html. 
vicio", que en determinados contextos podría tener connotaciones negativas tanto desde el punto de vista social como educativo.

Somos conscientes de que el término "solidaridad" no tiene las mismas implicancias en todas las lenguas, por ello intentaremos aclarar brevemente el alcance con que usamos el término en el siguiente cuadro:

Cuadro 1: Servicio vertical y solidaridad horizonta $2^{23}$

\begin{tabular}{|l|l|}
\hline \multicolumn{1}{|c|}{ Servicio "vertical" } & \multicolumn{1}{c|}{ Solidaridad "horizontal" } \\
\hline Dar-Hacer "para" & $\begin{array}{l}\text { Compartir-Hacer "con", co-protagonismo- } \\
\text { Reciprocidad }\end{array}$ \\
\hline $\begin{array}{l}\text { Altruísmo autocentrado: "Me hace } \\
\text { sentir bien" }\end{array}$ & Empatía, vínculos prosociales \\
\hline Paternalismo & Fraternidad \\
\hline Clientelismo & Empoderamiento \\
\hline $\begin{array}{l}\text { Imparte conocimientos y directivas desde } \\
\text { una posición de superioridad }\end{array}$ & $\begin{array}{l}\text { Intercambio y construcción conjunta } \\
\text { de saberes }\end{array}$ \\
\hline $\begin{array}{l}\text { Reproducción aún involuntaria } \\
\text { de situaciones de injusticia y de los ciclos } \\
\text { de pobreza y exclusión }\end{array}$ & $\begin{array}{l}\text { Reconocimiento de derechos, búsqueda } \\
\text { de equidad y justicia. }\end{array}$ \\
\hline
\end{tabular}

\subsection{El amor como sentido del proceso educativo}

En la educación tradicional, se presenta habitualmente a la adquisición de conocimientos como un fin en sí mismo. Para muchos estudiantes, todo el sentido de su paso por una institución educativa se reduce a la adquisición de un diploma, que esperan contribuya a la obtención de empleo y al acceso a una mejor situación económica ${ }^{24}$.

${ }^{23}$ CLAYSS, Service-Learning in Central and Eastern Europe. Handbook for Engaged Teachers and Students (Buenos Aires: CLAYSS, 2017), 18-24, http://www.clayss.org.ar/04_ publicaciones/SL-EE_nov17.pdf.

${ }^{24}$ Francisco, "Discurso a la comunidad de la Universidad Católica portuguesa con ocasión del 50 aniversario de su fundación", 26.10.2017, http://w2.vatican.va/content/francesco/es/ speeches/2017/october/documents/papa-francesco_20171026_universita-cattolica-portoghese. html 
La propuesta de sentido del aprendizaje-servicio, en cambio, es "aprender para poder servir a los otros". "Para algunas Universidades el objeto de su existencia es la excelencia académica. Nosotros consideramos que la razón de nuestra existencia es el servicio a la gente y la excelencia académica su mejor instrumento" 25 .

Como señalan Furco y otros ${ }^{26}$, es justamente el sentido solidario de los proyectos de aprendizaje-servicio lo que motiva a los estudiantes e impulsa su interés por aprender en mayor medida que en el marco de otras pedagogías más tradicionales. Esta propuesta de sentido para la educación más allá de sí está también en el centro del mensaje de Chiara Lubich a los jóvenes del movimiento de los Focolares, los "Gen". En uno de sus primeros mensajes les decía: "Si descuidas tus estudios, eres un irresponsable. Si estudias por la nota, eres un orgulloso. Si estudias por amor a Dios y a los demás, entonces encontraste el equilibrio" 27.

El estrecho vínculo entre el amor concreto al prójimo y la luz de una mayor comprensión espiritual y humana se sintetizaba en los primeros años del Movimiento Gen en la frase "Ama y comprenderás". Esta afirmación, que podría considerarse válida sólo para la vida espiritual, lo es también desde el punto de vista educativo para la pedagogía del aprendizaje-servicio. A través de sus proyectos solidarios con la comunidad, los estudiantes pueden aplicar y desarrollar saberes y competencias, expresar sus inteligencias múltiples, fortalecer actitudes prosociales y de compromiso ciudadano ${ }^{28}$.

Chiara Lubich subrayaba la importancia formativa de las prácticas solidarias para los jóvenes. En un discurso a jóvenes comprometidos en el movimiento Gen les decía en 1977:

Ustedes comprendieron la espiritualidad, pero a los que llegan recién ahora al Movimiento Gen no pueden ponerlos 24 horas sobre 24 a vivir solamente su dimensión espiritual. Ellos tienen necesidad de donarse [...] a los pobres, a los enfermos, etc.; hay que ofrecerles una tarea inmediata. [...] amando, ellos en-

${ }^{25}$ Docentes y estudiantes de la Facultad de Medicina de la Universidad de Tucumán, Argentina. En: Ministerio de Educación, Ciencia y Tecnología, Experiencias ganadoras del Premio Presidencial "Prácticas Solidarias en Educación Superior" 2004 (República Argentina: MECyT, 2006), 11.

${ }^{26}$ Furco, "Impacto".

27 Chiara Lubich, 100 Pensamientos (Buenos Aires: Ciudad Nueva, 1989), 16.

${ }^{28}$ Eyler, Giles, Where's the learning; Billig, "Heads"; Billig, "Lessons"; Jacoby, Servicelearning. 
contrarán la luz y con la luz - [...] que es Dios - amarán todavía más. Por lo tanto, la palabra de orden es: dar la vida por la propia gente ${ }^{29}$.

De hecho, parte importante de la praxis formativa de Lubich hacia los jóvenes del movimiento era la frecuente organización de acciones internacionales de solidaridad, como la Operación África ${ }^{30}$, junto con el estímulo al desarrollo de obras sociales locales.

El amor concreto, el "mandamiento nuevo" de dar la vida por los hermanos está justamente en el centro del mensaje cristiano y del carisma de la unidad.

En su primer libro de Meditaciones, Lubich afirma que si como estudiantes supiéramos las preguntas qué nos van a tomar en el último examen del año, nos consideraríamos muy afortunados y las estudiaríamos a fondo. Y nos recuerda que El Maestro ya nos dejó las preguntas que nos hará al final de la vida:

La vida es una prueba, y al final de ella también hay que superar un examen.

Pero el amor infinito de Dios ya le ha hecho saber al hombre cuáles serán las preguntas: "Tuve hambre y me diste de comer, tuve sed y me diste de beber". Las obras de misericordia serán materia de examen, esas obras en las cuales Dios ve si se lo ha amado verdaderamente, habiéndolo servido en el hermano ${ }^{31}$.

A partir de esta premisa, podría afirmarse que desde una perspectiva cristiana el aprendizaje-servicio es una pedagogía que contribuye a la preparación para el "examen final".

En términos de las grandes religiones implicaría el aprendizaje para vivir según "la regla de oro" 32 de hacer al prójimo lo que quisiéramos que nos hicieran a nosotros ${ }^{33}$. Aún desde una perspectiva no religiosa, podría decirse que

${ }^{29}$ Chiara Lubich, Coloquios con los Gen desde 1966 hasta 2000 (Buenos Aires: Ciudad Nueva, 2005), 348.

${ }^{30}$ Cf. http://www.focolare.org/news/2009/01/10/per-colmare-un-debito-nasce-1\%e2\% $80 \% 99$ operazione-africa/

${ }^{31}$ Chiara Lubich, Meditaciones (Buenos Aires: Ciudad Nueva, 1971), 89-90.

32 Bill Puka, "The Golden Rule", in: The Internet Encyclopedia of Philosophy, ed. James Fieser, Bradley Dowden, http://www.iep.utm.edu/goldrule/; cf. La regola d'oro come etica universale, ed. Carmelo Vigna, Susy Zandaro (Milano: Vita e Pensiero, 2005).

${ }^{33}$ Chiara Lubich, "Discurso a los jóvenes reunidos delante del Coliseo de Roma para el Supercongreso Gen”, Roma, 26.05.2002, http://www.focolare.org/es/news/2016/01/03/laregola-doro-come-via-per-la-costruzione-della-fratellanza-e-della-pace/. 
lo más esencial del ser humano es su capacidad de amar intencionadamente a los demás, de aprender lo que Erich Fromm llamaba "el arte de amar"34. En este sentido, el aprendizaje-servicio podría considerarse una suerte de didáctica para el aprendizaje de cómo amar concretamente a los demás.

Central a las enseñanzas de Chiara Lubich es justamente "el arte de amar" ${ }^{\prime 5}$. Lubich lo resumía en seis puntos fundamentales, que pueden leerse como una potente base de reflexión sobre el tipo de vínculos interpersonales y sociales que se establecen en los proyectos de aprendizaje-servicio solidario $^{36}$.

Si bien el tema merecería mayor profundización que aquí no cabe desarrollar, una primera aproximación puede encontrarse en el siguiente cuadro:

Cuadro 2: El "arte de amar" y la pedagogía del aprendizaje-servicio solidario

\begin{tabular}{|c|c|}
\hline El "arte de amar"38 & Aprendizaje-servicio solidario (AYSS) \\
\hline $\begin{array}{l}\text { 1. "Amar a todos": implica vivir } \\
\text { la fraternidad universal y estar } \\
\text { dispuestos a servir a todos ( } \operatorname{Lc} 22,27) \text {. }\end{array}$ & $\begin{array}{l}\text { Las prácticas de AYSS contribuyen } \\
\text { a descentrarse de sí y a abrir la institución } \\
\text { educativa hacia el territorio, para salir } \\
\text { al encuentro de problemas que afectan } \\
\text { al conjunto de una comunidad }{ }^{39} \text {. }\end{array}$ \\
\hline $\begin{array}{l}\text { 2. "Ser los primeros en amar": tomando } \\
\text { la iniciativa como María, que "partió } \\
\text { sin demora" a atender a su prima } \\
\text { Isabel (Lc. 1, 39); gratuitamente, } \\
\text { sin esperar recompensa. }\end{array}$ & 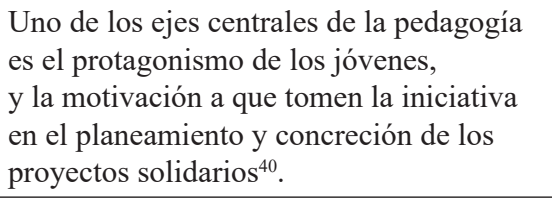 \\
\hline $\begin{array}{l}\text { 3. "Amar como a uno mismo": "Hagan } \\
\text { por los demás lo que quieren que los } \\
\text { hombres hagan por ustedes". } \\
\text { (Lc. } 7,31) \text {. }\end{array}$ & $\begin{array}{l}\text { Las buenas prácticas de AYSS contribuyen } \\
\text { a la formación de actitudes prosociales }{ }^{41} \text {, } \\
\text { superando actitudes egocéntricas y el } \\
\text { desinterés por el bien común, así como }\end{array}$ \\
\hline
\end{tabular}

${ }^{34}$ Erich Fromm, El arte de amar (Buenos Aires: Paidós, 2000).

${ }^{35}$ Chiara Lubich, El Arte de amar (Buenos Aires: Ciudad Nueva, 2007).

36 del Campo, La educación.

${ }^{37}$ Lubich, El Arte de amar.

${ }^{38}$ CLAYSS, Escuelas para el encuentro. Cómo desarrollar un proyecto de aprendizaje-servicio (Buenos Aires: CLAYSS, 2017), 47; http://www.clayss.org.ar/04_publicaciones/ Manual_CLAYSS_Scholas.pdf.

${ }^{39}$ del Campo, Protagonismo.

${ }^{40}$ Donald Eberly, Roberto Roche Olivar, "Aprendizaje-servicio y prosocialidad”, en: CLAYSS, Aprender sirve, servir enseña (Buenos Aires: CLAYSS, 2002), 33-48. 
Cuadro 2: El "arte de amar” y la pedagogía del aprendizaje-servicio solidario

\begin{tabular}{|c|c|}
\hline \multirow[t]{2}{*}{ El "arte de amar" } & Aprendizaje-servicio solidario (AYSS) \\
\hline & $\begin{array}{l}\text { la endogamia y desinterés por el entorno } \\
\text { social a veces presentes en las instituciones } \\
\text { educativas. }\end{array}$ \\
\hline $\begin{array}{l}\text { 4. "Haciéndose uno": como lo expresa } \\
\text { San Pablo "Me hice todo para todos" } \\
\text { (1Cor 9,22). amar haciendo propias } \\
\text { las necesidades y esperanzas de los } \\
\text { demás. }\end{array}$ & $\begin{array}{l}\text { La pedagogía del AYSS alienta el } \\
\text { desarrollo de la capacidad de empatía }{ }^{42} \text {, } \\
\text { y pone el acento en la importancia } \\
\text { de definir los objetivos de los proyectos } \\
\text { a través de diagnósticos participativos } \\
\text { y de la adecuada escucha y empatía con } \\
\text { los actores comunitarios, tanto a nivel } \\
\text { de las relaciones interpersonales como } \\
\text { inter-institucionales }{ }^{43} \text {. }\end{array}$ \\
\hline $\begin{array}{l}\text { 5. „Amar a Jesús en cada uno: } \\
\text { "Aquello que hiciste al más pequeño, } \\
\text { me lo hiciste a mí" (Mt 25,40). }\end{array}$ & $\begin{array}{l}\text { Si bien la pedagogía del AYSS es válida en } \\
\text { contextos de educación laica y de muchas } \\
\text { convicciones filosóficas y religiosas } \\
\text { diversas, en el contexto de la educación } \\
\text { cristiana las prácticas educativas solidarias } \\
\text { permiten reflexionar y experimentar } \\
\text { fuertemente la presencia de Jesús en cada } \\
\text { miembro de la comunidad atendida, y el } \\
\text { aprendizaje concreto del amor al prójimo } \\
\text { "con obras y de verdad" }(1 \mathrm{Jn} 3,18)^{44} \text {. }\end{array}$ \\
\hline $\begin{array}{l}\text { 6. "Amor recíproco": Con la medida } \\
\text { de Jesús: "ámense... como yo los he } \\
\text { amado" (Jn 15,12-13). El "dar la vida } \\
\text { por los amigos" (Jn 15,12-13) tarde } \\
\text { o temprano genera la reciprocidad, } \\
\text { y el amor recíproco atrae la presencia }\end{array}$ & $\begin{array}{l}\text { El AYSS constituye una forma de } \\
\text { aprendizaje colaborativo no sólo entre } \\
\text { los estudiantes, sino entre la institución } \\
\text { educativa y los actores comunitarios. } \\
\text { Propone una "solidaridad horizontal" } 45 \\
\text { y la producción de conocimiento como }\end{array}$ \\
\hline
\end{tabular}

${ }^{41}$ Laura Campo, "El aprendizaje servicio en la universidad como propuesta pedagógica", en: Aprendizaje servicio y responsabilidad social de las universidades, coord. Miquel Martínez Martín (Barcelona: Octaedro, 2008), 81-91; Roser Batlle, "Aprendizaje Servicio para una Educación Empática", https://youtu.be/ZLFbydlaC0E.

${ }^{42}$ Tapia, Bridi, Maidana, Rial, El compromiso, 73-81.

43 Francisco, "Mensaje I Jornada Mundial de los Pobres", 19.11.2017, https://w2.vatican. $\mathrm{va} /$ content/francesco/es/messages/poveri/documents/papa-francesco_20170613_messaggio-i-giornatamondiale-poveri-2017.html.

${ }^{44}$ Antonio Maria Baggio, "Se la politica riscopre la fraternità", Avvenire (Roma, 05.04. 2007). 
Cuadro 2: El "arte de amar" y la pedagogía del aprendizaje-servicio solidario

\begin{tabular}{|l|l|}
\hline \multicolumn{1}{|c|}{ El "arte de amar" } & \multicolumn{1}{|c|}{ Aprendizaje-servicio solidario (AYSS) } \\
\hline $\begin{array}{l}\text { de Jesús en medio de la comunidad } \\
\text { unida en su nombre (Mt. 18,20). }\end{array}$ & un don recíproco, en el que el conocimiento \\
Él presente en medio de los que se & y del diálogo respetuoso sabiduría de las \\
aman, puede realizar su "testamento" & culturas populares y originarias en un \\
de la última cena: "Que todos sean & "círculo virtuoso". \\
uno: como tú, Padre, estás en mí & En el contexto de una educación cristiana, \\
y yo en ti, que también ellos sean uno & el AYSS permite generar vínculos de \\
en nosotros, para que el mundo crea". & unidad entre docentes, estudiantes y otros \\
& miembros de la comunidad, abiertos a "dar \\
& la vida por la propia gente" a través de \\
acciones concretas, dando así testimonio \\
ante muchos y construyendo "fragmentos \\
de fraternidad" en donde se encuentran.
\end{tabular}

\subsection{Un amor que planifica y transforma la sociedad}

En uno de sus mensajes a los niños y adolescentes miembros del Movimiento, Chiara Lubich les decía que era necesario "tomar las medidas" de su ciudad para transformarla, y que si amaban a Dios "Una ciudad no basta" 45. El análisis de este escrito desde la perspectiva del AYSS merecería un artículo completo.

Baste aquí señalar que las herramientas metodológicas desarrolladas por el aprendizaje-servicio - como el "itinerario" para los proyectos ${ }^{46}$ - parten, como el mensaje de Lubich, de asumir que aún desde muy pequeños "todos somos candidatos" a transformar el mundo, y que para hacerlo no bastan las buenas intenciones, sino que se necesita un amor inteligente que sepa plantearse objetivos y planificar cómo alcanzarlos.

Desde ese punto de vista, la pedagogía del AYSS es una poderosa herramienta que ya utilizan miles de instituciones educativas religiosas para articular en el mismo proyecto el conocimiento académico, el desarrollo de

45 Chiara Lubich, Scritti Spirituali (I) (Roma: Città Nuova, 1978); Chiara Lubich, Mensaje a los jóvenes (Roma, 2006), http://www.focolare.org/es/news/2006/05/02/una-citta-non-basta/

${ }^{46}$ CLAYSS, Escuelas, 56-86; Ministerio de Educación, Itinerario y herramientas para desarrollar un proyecto de aprendizaje-servicio (Buenos Aires: Ministerio de Educación de la Nación, 2015) https://www.argentina.gob.ar/sites/default/files/edusol-itinerario-y-herramientasedicion-2014-5901e6a68e7dd.pdf 
competencias y la formación humana y religiosa ${ }^{47}$. En las palabras del Papa Francisco, permite unir "los lenguajes de la cabeza, el corazón y las manos"48.

\section{Conclusiones}

Por razones de espacio, no podemos desarrollar aquí algunos otros conceptos básicos en cuanto a los fundamentos teóricos, las herramientas y metodologías propias de la pedagogía del aprendizaje-servicio solidario, y otras vinculaciones con el carisma de Chiara Lubich que podrían señalarse, pero los elementos presentados pueden mostrar suficientemente sus numerosos puntos en contacto.

Si bien la influencia del pensamiento de Lubich sobre la pedagogía del aprendizaje-servicio es relativamente reciente y aparece explicitada casi exclusivamente en la bibliografía en lengua española e italiana, entendemos que la afinidad entre el carisma de Lubich y el aprendizaje-servicio abre posibilidades para ulteriores estudios y profundizaciones en este sentido.

Como nos exhortaba el Papa Francisco, "No vamos a cambiar el mundo si no cambiamos la educación" ${ }^{\text {" }}$. Creemos que las prácticas que articulan aprendizaje y solidaridad permiten apuntar a los dos objetivos al mismo tiempo, y esperamos que estas breves páginas alienten el desarrollo de proyectos de aprendizaje-servicio solidario en Polonia como en otras partes del mundo.

\section{Referencias}

Baggio, Antonio Maria. "Se la politica riscopre la fraternità". Avvenire, Roma, 05.04. 2007.

Batlle, Roser. "Aprendizaje Servicio para una Educación Empática”. https://youtu. be/ZLFbydlaC0E.

${ }^{47}$ Tapia, Bridi, Maidana, Rial, El compromiso; Arantzazu Martinez-Odria, Isabel Gómez Villalba, Aprendizaje-servicio (educar para el encuentro (Madrid, Editorial KHAK, 2018).

${ }^{48}$ Francisco, "Discorso ai partecipanti al Congresso Mondiale promosso dalla Congregazione per l'Educazione Cattolica”, 21.10.2015, http://w2.vatican.va/content/francesco/it/ speeches/2015/november/documents/papa-francesco_20151121_congresso-educazione-cattolica.html.

49 Francisco, "Mensaje al Congreso de Scholas", 5.02.2015, http://w2.vatican.va/content/ francesco/es/speeches/2015/february/documents/papa-francesco_20150205_scholas-occurrentes.html 
Batlle, Roser. El aprendizaje-servicio en España: el contagio de una revolución pedagógica necesaria. Madrid: PPC, 2013.

Billig, Shelley. "Lessons from Reseach on Teaching and Learning: Service-learning as Effective Instruction". En: Growing to Greatness: The State of Service Learning Project 2004 Report. St. Paul: NYLC \& State Farm, 2006.

Billig, Shelley. "Heads, Hearts, and Hands: The Research on K-12 Service Learning". En: Growing to Greatness: The State of Service Learning Project 2004 Report. St. Paul: NYLC \& State Farm, 2004.

Brown, Danika M. Pulling it Together: A Method for Developing Service-Learning and Community Partnerships Based in Critical Pedagogy. Washington DC: National Service Fellow Research, 2001.

Campo, Laura. "El aprendizaje servicio en la universidad como propuesta pedagógica". En: Aprendizaje servicio y responsabilidad social de las universidades, coord. Miquel Martínez Martín, 81-91. Barcelona: Octaedro, 2008.

Chanes, Silvia, Graciana Gaona, María Nieves Tapia. "Caminos de solidaridad”. Conferencia Internacional Católica del Guidismo. Documentos pedagógicos. 2006. http://www.clayss.org.ar/04_publicaciones/Caminosdesolidaridad-esp.pdf.

CLAYSS (Centro Latinoamericano de Aprendizaje y Servicio Solidario-Scholas). Escuelas para el encuentro. Cómo desarrollar un proyecto de aprendizaje-servicio. Buenos Aires: CLAYSS, 2017. http://www.clayss.org.ar/04_publicaciones/Manual_CLAYSS_Scholas.pdf.

CLAYSS. Guía para desarrollar proyectos de aprendizaje-servicio solidario. Edición Perú. Lima: CLAYSS, 2018. http://www.clayss.org.ar/04_publicaciones/ Manual_Peru_para_web.pdf.

CLAYSS. Programa de aprendizaje-servicio como politica educativa nacional en Uruguay. Evaluación de resultados. Buenos Aires: CLAYSS-Porticus-Zigla, 2017.

CLAYSS. Service-Learning in Central and Eastern Europe. Handbookfor Engaged Teachers and Students. Buenos Aires: CLAYSS, 2017. http://www.clayss.org. ar/04_publicaciones/SL-EE_nov17.pdf

Congregation for Catholic Education. Educating today and tomorrow. A renewing passion. Vatcan City, 2014.

Deans, Thomas. "Service-Learning in Two Keys: Paulo Freire’s Critical Pedagogy in Relation to John Dewey's Pragmatism". Michigan Journal of Community Service Learning 6 (1999): 15-29.

Del Campo, Graciela. La educación solidaria en el marco de la nueva evangelización de lo social. Tesis de Maestría en Doctrina Social de la Iglesia. Madrid: Universidad Pontificia de Salamanca, 2015. Texto inédito.

Del Campo, Graciela. Protagonismo estudiantil solidario: alternativas de participación ciudadana y compromiso político desde la escuela. La experiencia de 
estudiantes que participan de Proyectos educativos solidarios. Ponencia presentada en: Universidad Nacional de Avellaneda, Primeras Jornadas de Educación y Política, 24 de septiembre de 2013.

Delors, Jacques. La educación encierra un tesoro. Buenos Aires: Santillana, Ediciones UNESCO, 1996.

Eberly, Donald J. National Service: A Promise to Keep. Rochester, NY: John Alden Books, 1988.

Eberly, Donald, Roberto Roche Olivar. "Aprendizaje-servicio y prosocialidad". En: CLAYSS, Aprender sirve, servir enseña, 33-48. Buenos Aires: CLAYSS, 2002.

Eyler, Janet, Dwight E. Giles. Where's the learning in service-learning? San Francisco: Jossey-Bass Publishers, 1999.

Francisco. "Discorso ai partecipanti al Congresso Mondiale promosso dalla Congregazione per l'Educazione Cattolica", 21.10.2015. http://w2.vatican.va/content/ francesco/it/speeches/2015/november/documents/papa-francesco_20151121_ congresso-educazione-cattolica.html

Francisco. "Discurso a la comunidad de la Universidad Católica portuguesa con ocasión del 50 aniversario de su fundación”, 26.10.2017. http://w2.vatican.va/ content/francesco/es/speeches/2017/october/documents/papa-francesco_20171 026_universita-cattolica-portoghese.html.

Francisco. "Mensaje al Congreso de Scholas", 5.02.2015. http://w2.vatican.va/content/francesco/es/speeches/2015/february/documents/papa-francesco_201502 05_scholas-occurrentes.html.

Francisco. "Mensaje I Jornada Mundial de los Pobres", 19.10.2017. https://w2.vatican.va/content/francesco/es/messages/poveri/documents/papa-francesco_2017 0613_messaggio-i-giornatamondiale-poveri-2017.html

Freire, Paulo, Myles Horton. We Make the Road by Walking: Conversations on Education and Social Change. Philadelphia: Temple University Press, 1991.

Fromm, Erich. El arte de amar. Buenos Aires: Paidós, 2000.

Furco, Andrew. "Impacto de los proyectos de aprendizaje-servicio". En: Aprendizaje y servicio solidario en la Educación Superior y en los sistemas educativos latinoamericanos. Actas del 7mo. Seminario Internacional "Aprendizaje y Servicio Solidario", 19-26. República Argentina: Ministerio de Educación, 2005. http://www.clayss.org.ar/seminario/anteriores/actas/2005_Actas7.pdf.

Furco, Andrew. "The community as a resource for learning: an analysis of academic service-learning in primary and secondary education". En: The Nature of Learning. Using Research to Inspire Practice, ed. Hanna Dumont, David Instance, Francisco Benavides. Paris: Organisation for Economic Co-operation and Development, Centre for Educational Research and Innovation, 2010. 
González, Alba, Pablo Elicegui. "El impacto de los proyectos de aprendizaje-servicio en la calidad educativa. Reflexiones en torno a ocho experiencias". En: Ministerio de Educación, Ciencia y Tecnología, Aprendizaje y servicio solidario. Actas del 5to. y 6to. Seminario Internacional “Aprendizaje y servicio solidario”, 188-206. República Argentina, 2004.

International Association for National Youth Service. Actas de la $6^{a}$ Conferencia Global/Procedures of the VI Global Conference. Buenos Aires: CLAYSS, 2002.

Jacoby, Barbara. Service-learning Essentials. Questions, Answers, and Lessons Learned. San Francisco: Jossey Bass, 2015.

La regola d'oro come etica universale, ed. Carmelo Vigna, Susy Zandaro. Milano: Vita e Pensiero, 2005.

Lubich, Chiara. "Discurso a los jóvenes en Loppiano", FI, Italia, 2.05.2006. http:// www.focolare.org/es/news/2006/05/02/una-citta-non-basta/

Lubich, Chiara. "Discurso a los jóvenes reunidos delante del Coliseo de Roma para el Supercongreso Gen”, Roma, 26.05.2002. http://www.focolare.org/es/news/ 2016/01/03/la-regola-doro-come-via-per-la-costruzione-della-fratellanza-edella-pace/.

Lubich, Chiara. 100 Pensamientos. Buenos Aires: Ciudad Nueva, 1989.

Lubich, Chiara. Coloquios con los Gen desde 1966 hasta 2000. Buenos Aires: Ciudad Nueva, 2005.

Lubich, Chiara. El Arte de amar. Buenos Aires: Ciudad Nueva, 2007.

Lubich, Chiara. Meditaciones. Buenos Aires: Ciudad Nueva, 1971.

Lubich, Chiara. Mensaje a los jóvenes. Roma, 2006.

Lubich, Chiara. Scritti Spirituali (I). Roma: Città Nuova, 1978.

Lubich, Chiara. Una cultura nueva para una nueva sociedad. Doctorados honoris causa-Congresos Internacionales (1996-2001). Buenos Aires: Ciudad Nueva, 2003.

Mardones, Rodrigo. Educación y fraternidad: Un principio para la formación ciudadana y la convivencia democrática. Buenos Aires: Ciudad Nueva, 2013.

Martín García, Xus. Per a una pedagogía del servei. Treball per projectes i APS. Barcelona: Minyons Escoltes Guies Sant Jordi de Catalunya, 2012.

Martinez-Odria, Arantzazu, Isabel Gómez Villalba. Aprendizaje-servicio (educar para el encuentro). Madrid: Editorial KHAK, 2018.

Ministerio de Educación, Ciencia y Tecnología. Experiencias ganadoras del Premio Presidencial "Prácticas Solidarias en Educación Superior” 2004. República Argentina: MECyT, 2006.

Ministerio de Educación. Experiencias ganadoras del Premio Presidencial "Escuelas Solidarias” 2009. República Argentina: Ministerio de Educación, 2010. 
Moore McBride, Amanda, Carlos Benitez, Michael Sherraden, Danso Kwofie, Beatriz Castaño, Lissa Johnson, Maury Mendenhall, Erica Sulever, Erdenechimeg Tserendorj, Jenny Brav, Lacey Clark. The forms and Nature of Civic Service: A Global Assessment. Washington: University in St. Louis, Global Service Institute, Center for Social Development, 2003.

Movimento dei Focolari. "Sei tappe per un obietivo". Teens (2018).

Ochoa, Enrique. "Aprendizaje-servicio en América Latina. Apuntes sobre pasado y presente". TZHOECOEN 5 (2010): 108-125.

Office of Service-Learning, Lingnan University. Annual report 2011-2012. Hong Kong: Lignan University, 2012.

PaSo Joven: Participación Solidaria para América Latina. "Manual de formación de formadores en aprendizaje-servicio y servicio juvenil". Buenos Aires: BIDSES-CLAYSS-ALIANZAONG-CEBOFIL,2004.http://www.clayss.org.ar/paso joven/index.htm

Puka, Bill. "The Golden Rule". En: The Internet Encyclopedia of Philosophy, ed. James Fieser, Bradley Dowden. http:/www.iep.utm.edu/goldrule/.

Service-learning in South Africa, ed. Ruksana Osman, Nadine Petersen. Cape Town: Oxford University Press, 2013.

Tapia, María Nieves, Enrique Ochoa. "Legislación y normativa latinoamericana sobre servicio comunitario estudiantil y aprendizaje-servicio". En: Actas de la III Jornada de investigadores sobre aprendizaje-servicio, comp. María Alejandra Herrero, María Nieves Tapia, 91-96. Buenos Aires: CLAYSS-Red Iberoamericana de aprendizaje-servicio, 2015.

Tapia, María Nieves, Gerardo Bridi, María Paula Maidana, Sergio Rial. El compromiso social como pedagogía. Aprendizaje y solidaridad en la escuela. Bogotá: CELAM, 2015.

Tapia, María Nieves. "Aprendizaje-servicio, un movimiento pedagógico mundial”. En: Aprendizaje-servicio (ApS): claves para su desarrollo en la Universidad, coord. Laura Rubio, Anna Escofet, 55-68. Barcelona: Octaedro-ICE, 2017.

Tapia, María Nieves. Aprendizaje y servicio solidario en el sistema educativo y las organizaciones juveniles. Buenos Aires: Ciudad Nueva, 2006.

Tapia, María Nieves. La solidaridad como pedagogía. Buenos Aires: Ciudad Nueva, 2000.

Titlebaum, Peter, Gabrielle Williamson, Corinne Daprano, Janine Baer, Jayne Brahler. Annotated History of Service Learning 1862-2002. Dayton, OH: University of Dayton, May 2004.

UKM, National University of Malaysia. Strengthening Community Engagement. Nurturing Caring Citizens. Kuala Lumpur: UKM, 2012. 
